\title{
OPEN Optimizing surgical outcome of auricular keloid with a novel multimodal approach
}

\author{
Yi-Teng Hung ${ }^{1,2,3}$, Shih-Min Lin ${ }^{3,4}$, I-Shiang Tzeng ${ }^{5}$ \& Chau Yee $\mathrm{Ng}^{1,2,3 凶}$
}

Various treatments are available for auricular keloids, but none has an absolute advantage. A practical and safe therapy to optimize the surgical outcome for auricular keloids is needed. We adopted a multimodal treatment of surgical enucleation, core fillet flap reconstruction, intraoperative corticosteroid injection, and immediate postoperative radiotherapy. There were no routine intralesional corticosteroid injections during follow-up. Keloid recurrences, complications, and risk factors for recurrences were analyzed. The outcome was compared with other published literatures. 45 auricular keloids were included in this study. $85.7 \%$ were female with an average age of $27.1 \pm 7.5$ years, and averaged size was $1.8 \times 1.2 \pm 0.9 \times 0.6 \mathrm{~cm} .71 .1 \%$ were located at ear helix with 28.9\% at the ear lobe. Nine keloids were classified as Chang-Park classification type I, 30 for type II, two for type III, and four for IV. The average radiation dosage was $1578.6 \mathrm{cGy}$. The recurrence rate was $6.7 \%$ at an average 24.1 -month follow-up. There were no complications of surgery, radiotherapy, and intralesional corticosteroid injection. Our recurrence rate was lower than those in mono-adjuvant therapies of intraoperative corticosteroid injection or radiotherapy. This one-session multimodal approach optimizes treating auricular keloids with a low recurrence rate and minimal post-radiation and long-term corticosteroid injection-related complications.

Keloid is a wound healing complication with a continually growing scar following minor trauma, infection, burn, or inflammation ${ }^{1}$, and is characterized by aberrant growth of scar tissue extending beyond the previous wound margins. The ear is a common location of keloid formation, usually caused by ear piercing. Patients are often teenagers and young adults, whereby disfigured auricular keloids impose psychological stress and anxiety. Surgical excision is indicated for large, disfigured auricular keloids. However, surgical excision alone has a high recurrence rate up to $100 \%$, suggesting the importance of adjuvant therapy ${ }^{2}$. The postoperative adjuvant therapies consist of intralesional corticosteroid injection, radiation, pressure therapy, cryotherapy therapy, topical mitomycin C, topical imiquimod, and intralesional 5-fluorouracil ${ }^{3}$. Nevertheless, there has been no standard regimen for auricular keloids to achieve the goal of a low recurrence rate, minimal side effects, and significant aesthetic improvement of auricular contour. Many of these treatments require multiple postoperative session visits. Therefore, the establishment of optimal multimodal therapy for auricular keloids is critical. In this study, we presented our experience in treating auricular keloids with a multimodal approach in a single session composed of surgical enucleation, core fillet flap reconstruction, intraoperative intralesional corticosteroid injection, and immediate postoperative radiotherapy.

\section{Methods}

Patient collection. We retrospectively reviewed the electronic medical database and photographs of all patients receiving auricle keloid surgery from a tertiary medical center from December 2017 to May 2021. Patients who received a multimodal therapy composed of surgical enucleation, core fillet flap reconstruction, intraoperative intralesional injection, and immediate postoperative radiotherapy were included for analyses. Patients with incomplete medical records or photography were excluded from this study.

A total of 45 auricular keloids from 35 patients were included. The clinical characteristics, including age, sex, previous history of keloid treatment, size and location of the auricular keloid, postoperative wound healing, recurrence, and follow-up periods, were recorded. We classified the auricle keloids with the modified Chang-Park

${ }^{1}$ Department of Dermatology, Chang Gung Memorial Hospital Linkou Main Branch, Taoyuan, Taiwan. ${ }^{2}$ Department of Dermatologic Surgery, Chang Gung Memorial Hospital Linkou Main Branch, Taoyuan, Taiwan. ${ }^{3}$ School of Medicine, College of Medicine, Chang Gung University, Taoyuan, Taiwan. 'Department of Radiation Oncology, Chang Gung Memorial Hospital Linkou Main Branch, Taoyuan, Taiwan. ${ }^{5}$ Department of Statistics, National Taipei University, Taipei, Taiwan. ${ }^{\circledR}$ email: mdcharlene@gmail.com 
classification based on clinical photographs ${ }^{4}$. Modified Chang-Park classification of auricular keloids consists of type I (pedunculated type), type II (sessile type with a single nodular pattern), type III (sessile type with a multi-nodular pattern), type IV (buried type), and type V (mixed type) ${ }^{4}$.

Multimodal treatment protocol. Surgical method. The surgical method of enucleation and reconstruction with a core fillet flap was performed under local anesthesia (1\% lidocaine with 1:100,000 epinephrine). The overlying skin of the keloid was dissected from the underlying keloid mass as a core fillet flap. Subsequently, the keloid core mass was enucleated, and bleeding was meticulously controlled by electrocauterization. The fillet flap is an axial pedicle flap, first introduced in reconstructing traumatic amputation ${ }^{5}$, utilizing the "spare parts" from the adjacent tissue. We used this method to reconstruct the wound after keloidectomy ${ }^{6}$. The excessive part was trimmed meticulously, and the surgical margin was approximated with 6-0 nylon sutures.

Intraoperative intralesional corticosteroid injection. Immediately after excision, triamcinolone acetonide $(40 \mathrm{mg} / \mathrm{mL})$ at a pure concentration was injected into the surrounding dermal skin with the volume given proportional to incision length (range, $0.1-0.5 \mathrm{~mL}$ ). There were no postoperative injections during follow-up visits unless the patient has a sign of recurrence.

Radiotherapy. Keloids were irradiated with a 6-MeV electron beam of 15-18 Gy in three fractions by the linear accelerator (Varian Clinac ${ }^{\oplus} \mathrm{iX}$ ) performed at a $110-\mathrm{cm}$ source-to-skin distance. The first fraction was completed within postoperative 24 hours, and the remaining two fractions were performed in the consecutive four days. The scar regions after excision were positioned and confirmed by the radiation oncologist. A $0.5-\mathrm{cm}$ silicone bolus was used to improve the surface dose. The radiation field included the entire postoperative scar with a margin of $1 \mathrm{~cm}$. The field was shaped by a unique cone and $0.8-\mathrm{cm}$ customized cerebrum block.

Therapeutic effect evaluation. Recurrence was defined as the presence of hypertrophic scar raised above the level of the adjacent skin shown in the clinical photographs or medical records. We also extracted the records of the wound healing time, total follow-up period, and postoperative complications. We further analyzed the clinical characteristics of patients, type of auricular keloids, and radiation dosage to determine the risk factors for keloid recurrences.

Statistical analysis. Statistical analyses were performed with R software. The effect size calculated from a power analysis (Cohen's $d=0.9$ ) showed 42 . Continuous variables were described with means and standard deviations, while categorical variables were presented as numbers and percentages. The variables between recurrence and non-recurrence groups were analyzed with Student's T-test, Chi-square test, and Mann-Whitney U test. A $p$-value $<0.05$ was considered statistically significant.

Ethics and groups. This study was approved by the Chang Gung Medical Foundation Institutional Review Board (IRB no. 202100809B0). All methods were carried out in accordance with relevant guidelines and regulations. Informed consent was waived by Chang Gung Medical Foundation IRB.

\section{Results}

Clinical characteristics. The majority of patients were of Asian ethnicity, with only one Caucasian. The mean age was 27.1 years, ranging from 18 to 55 years. The location of keloids was divided into helix (including the cartilage part) and earlobe, with 32 keloids (71.1\%) and 13 keloids (28.9\%) for each, respectively (Table 1). The average dimension of these keloids (longitudinal length $\times$ horizontal width) was $1.8 \mathrm{~cm} \times 1.2 \mathrm{~cm}$, and the preoperative clinical photos were shown (Fig. 1a,b). There were 38 (84.4\%), eight (17.8\%), and four (8.9\%) keloids treated previously with intralesional corticosteroid injection, surgery, and cryotherapy, respectively. According to Chang-Park classification, nine keloids were classified as type I, 30 for type II, two for type III, four for IV, and none for type $\mathrm{V}$ (Table 1 ).

Therapeutic outcome. The preliminary result showed keloid recurrences occurred in 3 of 45 (6.7\%) (Table 2). One recurrence case was associated with pregnancy. The mean wound healing time was 2.4 weeks. There were no flap necrosis, surgical wound infection, or complications of corticosteroid injection and radiotherapy; only one case presented with seroma formation after 2.5 months, which subsided following incision and drainage. Clinical photographs of the postoperative auricular keloids taken during follow-up visits were shown (Fig. 1c,d).

Risk factors associated with recurrence. There were no significant differences in age, sex, previous treatment history, size, location at the ear, and type of Chang-Park classification among the auricular keloids with recurrences and without recurrences (Table 3). Radiation dosage was significantly higher (1584.6 cGy) in the auricular keloids without recurrences than that with recurrences (1500.0 cGy) $(p=0.001)$ (Table 3). Woundhealing time was similar in the keloids with recurrences and without recurrences. 


\begin{tabular}{|c|c|c|}
\hline & Mean $(\mathbf{N})$ & SD (\%) \\
\hline Age (years) & 27.1 & 7.5 \\
\hline \multicolumn{3}{|l|}{ Sex (patients) } \\
\hline $\mathrm{M}$ & 5 & 14.3 \\
\hline $\mathrm{F}$ & 30 & 85.7 \\
\hline \multicolumn{3}{|c|}{ Previous treatment (ears) } \\
\hline Surgery & 8 & 17.8 \\
\hline Intralesional injection & 38 & 84.4 \\
\hline Cryotherapy & 4 & 8.9 \\
\hline None & 7 & 15.6 \\
\hline \multicolumn{3}{|l|}{ Size $(\mathrm{cm})$} \\
\hline Length $\times$ width & $1.8 \times 1.2$ & $0.9 \times 0.6$ \\
\hline \multicolumn{3}{|c|}{ Location of ear keloid (ears) } \\
\hline Ear helix & 32 & 71.1 \\
\hline Ear lobe & 13 & 28.9 \\
\hline \multicolumn{3}{|c|}{ Chang-Park classification (ears) } \\
\hline I & 9 & 20 \\
\hline II & 30 & 66.7 \\
\hline III & 2 & 4.4 \\
\hline IV & 4 & 8.9 \\
\hline
\end{tabular}

Table 1. Characteristics of patients and auricular keloids. $F$ female, $M$ male, $N$ number, $S D$ standard deviation.
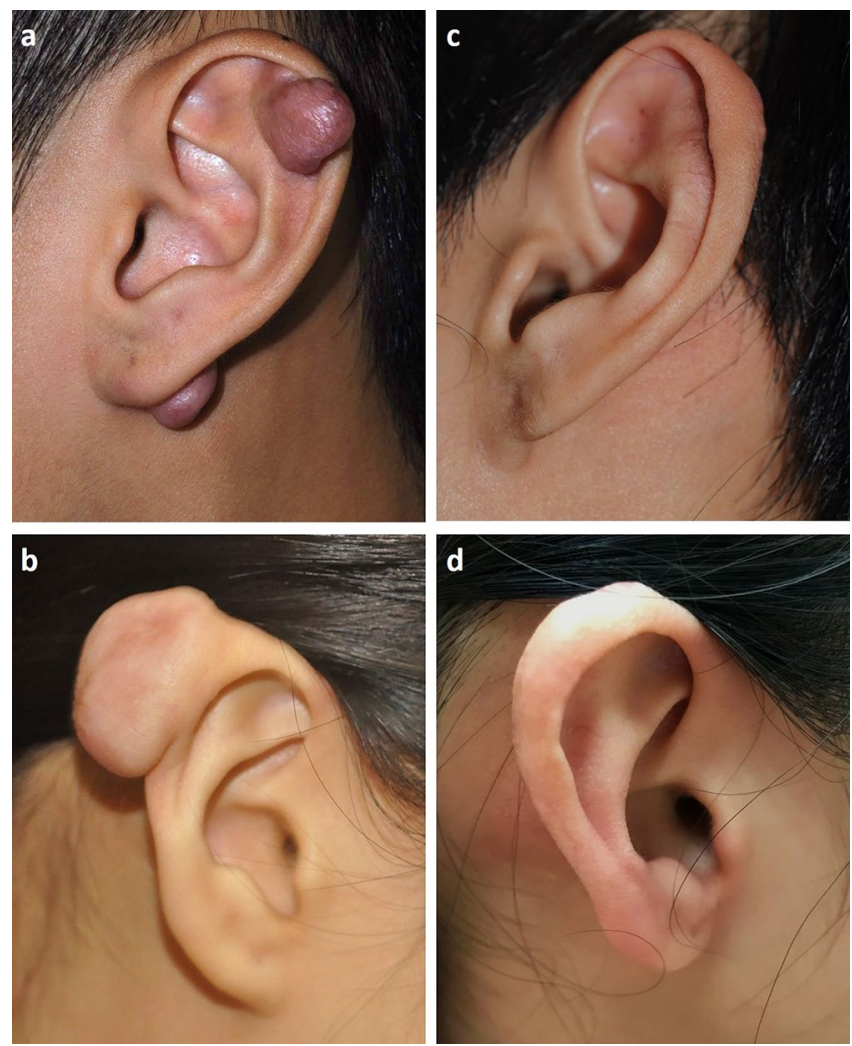

Figure 1. Clinical photographs of auricular keloids before and after treatment. (a) Two Chang-Park classification type I keloids located on the left ear helix and lobe. (b) Solitary large-sized Chang-Park classification type I keloid on the right ear helix. (c) The patient's appearance in (a) after 20 months of treatment. (d) The patient's appearance in (b) after 14 months of treatment. 


\begin{tabular}{|c|c|c|}
\hline & Mean $(\mathrm{N})$ & SD (\%) \\
\hline \multicolumn{3}{|l|}{ Radiation therapy } \\
\hline Dosage (cGY) & 1578.6 & 133.5 \\
\hline \multicolumn{3}{|l|}{ Interval (ears) } \\
\hline $0,1,2$ (day after OP) & 20 & 44.4 \\
\hline $0,1,4$ (day after OP) & 25 & 55.6 \\
\hline Wound healing (weeks) & 2.4 & 0.7 \\
\hline Recurrence (ears) & 3 & 6.7 \\
\hline Follow-up period (months) & 24.1 & 12.6 \\
\hline
\end{tabular}

Table 2. Treatment outcomes of auricular keloids. $N$ number, $O P$ operation, $S D$ standard deviation.

\begin{tabular}{|c|c|c|c|c|c|}
\hline & \multicolumn{2}{|c|}{$\begin{array}{l}\text { No recurrence } \\
(\mathrm{N}=42)\end{array}$} & \multicolumn{2}{|c|}{ Recurrence $(\mathrm{N}=3)$} & \multirow[b]{2}{*}{$p$-value } \\
\hline & Mean $(\mathrm{N})$ & SD (\%) & Mean $(\mathrm{N})$ & SD (\%) & \\
\hline Age (years) & 26.8 & 7.3 & 30.3 & 6.1 & 0.438 \\
\hline \multicolumn{6}{|l|}{ Sex (patients) } \\
\hline $\mathrm{M}$ & 5 & 11.9 & 0 & 0 & 0.447 \\
\hline $\mathrm{F}$ & 37 & 88.1 & 3 & 100 & \\
\hline \multicolumn{6}{|c|}{ Previous treatment (ears) } \\
\hline Surgery & 8 & 19.0 & 0 & 0 & 0.999 \\
\hline Intralesional injection & 35 & 83.3 & 3 & 100 & \\
\hline Cryotherapy & 2 & 4.8 & 2 & 66.7 & \\
\hline None & 7 & 16.7 & 0 & 0 & \\
\hline \multicolumn{6}{|l|}{ Size $(\mathrm{cm})$} \\
\hline Length $\times$ width & $1.9 \times 1.2$ & $0.9 \times 0.6$ & $1.5 \times 0.8$ & $0.9 \times 0.3$ & 0.472 \\
\hline \multicolumn{6}{|c|}{ Location of ear keloid (ears) } \\
\hline Ear helix & 30 & 71.4 & 2 & 66.7 & 0.999 \\
\hline Ear lobe & 12 & 28.6 & 1 & 33.3 & \\
\hline \multicolumn{6}{|c|}{ Chang-Park classification (ears) } \\
\hline I & 9 & 21.4 & 0 & 0 & 0.900 \\
\hline II & 27 & 64.3 & 3 & 100 & \\
\hline III & 2 & 4.8 & 0 & 0 & \\
\hline IV & 4 & 9.5 & 0 & 0 & \\
\hline \multicolumn{6}{|l|}{ Radiation therapy } \\
\hline Dosage (cGY) & 1584.6 & 136.8 & 1500 & 0 & 0.001 \\
\hline \multicolumn{6}{|l|}{ Interval (ears) } \\
\hline $0,1,2$ (day after OP) & 19 & 45.2 & 1 & 33.3 & 0.999 \\
\hline $0,1,4$ (day after OP) & 23 & 54.8 & 2 & 66.7 & \\
\hline
\end{tabular}

Table 3. Risk factors of keloid recurrences. $F$ female, $M$ male, $N$ number, $O P$ operation, $S D$ standard deviation.

\section{Discussion}

This novel multimodal approach in a single session composed of keloid enucleation, reconstruction with a core fillet flap, intraoperative intralesional corticosteroid injection, and immediate postoperative radiotherapy is an optimal regimen to treat auricular keloids with a low recurrence rate and few complications associated with radiotherapy and long-term corticosteroid injection.

Auricular keloids are challenging for surgical resection due to the preservation of the three-dimensional contour of the external ear and the scarcity of tissue laxity ${ }^{1}$. The recurrence rate of core enucleation with a fillet flap was lower than that of total excision, owing to the avoidance of high tension and distortion of anatomic structures. The nourished blood supply of fillet flaps ensured no event of flap necrosis in our study ${ }^{1}$. Nevertheless, adjuvant therapies were often needed due to a high recurrence rate of surgical resection alone, ranging between $45 \%$ and $100 \%{ }^{7}$. Intralesional corticosteroid injection and radiotherapy have been the most widely used regimens ${ }^{8}$. While the recurrence rate of radiotherapy was lower than that of intralesional corticosteroid injection ${ }^{9,10}$, irradiation could only damage the majority of keloid cells. Intralesional corticosteroid injections were needed to inhibit re-propagation of the residual irradiation-resistant keloid cells ${ }^{11}$. 


\begin{tabular}{|c|c|c|c|c|c|c|c|c|c|c|c|c|c|c|}
\hline & $\begin{array}{l}\text { Patient } \\
\text { (N) }\end{array}$ & $\begin{array}{l}\text { Keloid } \\
\text { (N) }\end{array}$ & Ethnicity & $\begin{array}{l}\text { Age } \\
\text { (years) }\end{array}$ & Sex & $\begin{array}{l}S_{i z e^{a}} \\
(\mathrm{~cm})\end{array}$ & Location & $\begin{array}{l}\text { Prior } \\
\text { OP }\end{array}$ & Prior IL & Surgery & $\begin{array}{l}\text { Scheduled } \\
\text { post-OP IL }\end{array}$ & $\begin{array}{l}\text { Recurrence } \\
\text { rate (keloid } \\
\mathrm{N} \text { ) }\end{array}$ & Follow-up & $\begin{array}{l}\text { Complication } \\
\text { (keloid N) }\end{array}$ \\
\hline $1^{14}$ & 64 & 92 & $\begin{array}{l}\text { African } \\
\text { Ameri- } \\
\text { can } \\
(83 \%) \\
\text { Cau- } \\
\text { casian } \\
(9 \%) \\
\text { Hispanic } \\
(8 \%)\end{array}$ & 28.3 & $\begin{array}{l}F(78 \%) \\
M(22 \%)\end{array}$ & 1.94 & NA & $\begin{array}{l}25 \% \\
(23 / 92)\end{array}$ & NA & $\begin{array}{l}\text { Total exci- } \\
\text { sion } \pm \text { flap }\end{array}$ & $\begin{array}{l}\text { Post-OP } \\
1 \text { st and 2nd } \\
\text { month }\end{array}$ & $\begin{array}{l}23.3 \% \\
(10 / 43)\end{array}$ & $\begin{array}{l}10.4 \text { years in } \\
43 \text { keloids }\end{array}$ & $\begin{array}{l}5.4 \%(5 / 92) ; \\
\text { depigmenta- } \\
\text { tion (1), scar } \\
\text { widening (1), } \\
\text { and wound } \\
\text { dehiscence (1) }\end{array}$ \\
\hline $2^{9}$ & 12 & 12 & $\begin{array}{l}\text { Black } \\
(67 \%) \\
\text { Cau- } \\
\text { casian } \\
(8 \%) \\
\text { Hispanic } \\
(25 \%)\end{array}$ & 29.4 & $\begin{array}{l}F(33 \%) \\
M(67 \%)\end{array}$ & 2.00 & $\begin{array}{l}\text { Lobe } \\
(100 \%)\end{array}$ & $\begin{array}{l}50 \% \\
(6 / 12)\end{array}$ & $\begin{array}{l}25 \% \\
(3 / 12)\end{array}$ & $\begin{array}{l}\text { Total exci- } \\
\text { sion }\end{array}$ & $\begin{array}{l}\text { Post-OP } \\
1 \text { st, 3rd, } \\
\text { and 5th } \\
\text { week }\end{array}$ & $33.3 \%(4 / 12)$ & 19 months & No \\
\hline $3^{6}$ & 15 & 21 & NA & 24.8 & $\mathrm{~F}(100 \%)$ & NA & $\begin{array}{l}\text { Lobe } \\
(71.4 \%) \\
\text { Helix } \\
(28.6 \%)\end{array}$ & $\begin{array}{l}13.3 \% \\
(2 / 15)\end{array}$ & $\begin{array}{l}20 \% \\
(3 / 15)\end{array}$ & $\begin{array}{l}\text { Enuclea- } \\
\text { tion + core } \\
\text { fillet flap }\end{array}$ & $\begin{array}{l}\text { Post- } \\
\text { OP 2nd } \\
\text { week and } \\
\text { monthly } \\
\text { dependent } \\
\text { on lesion }\end{array}$ & $9.5 \%(2 / 21)$ & 21.9 months & $\begin{array}{l}\text { Flap necrosis } \\
\text { and infec- } \\
\text { tion, abscess } \\
\text { after IL, and } \\
\text { neuroma after } \\
2^{\text {nd }} \text { OP }\end{array}$ \\
\hline Our & 35 & 45 & $\begin{array}{l}\text { Chinese } \\
(97.1 \%) \\
\text { Cau- } \\
\text { casian } \\
(2.9 \%)\end{array}$ & 27.1 & $\begin{array}{l}\mathrm{F} \\
(85.7 \%) \\
\mathrm{M} \\
(14.3 \%)\end{array}$ & 1.85 & $\begin{array}{l}\text { Lobe } \\
(71.1 \%) \\
\text { Helix } \\
(28.9 \%)\end{array}$ & $\begin{array}{l}17.8 \% \\
(8 / 45)\end{array}$ & $\begin{array}{l}84.4 \% \\
(38 / 45)\end{array}$ & $\begin{array}{l}\text { Enuclea- } \\
\text { tion + core } \\
\text { fillet flap }\end{array}$ & No & $6.7 \%(3 / 45)$ & 24.1 months & $\begin{array}{l}2.2 \%(1 / 45) \\
\text { seroma }(1)\end{array}$ \\
\hline
\end{tabular}

Table 4. Comparison with previous literatures adopting surgical excision with intraoperative [Triamcinolone

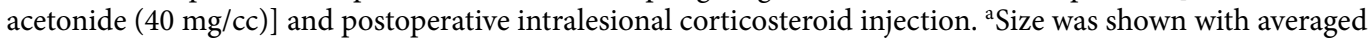
longitudinal length. $F$ female, $I L$ intralesional corticosteroid injection, $M$ male, $N$ number, $N A$ not available, $O P$ operation.

Corticosteroids can inhibit the migration of activated fibroblasts and induce vasoconstriction to reduce inflammatory responses ${ }^{12}$. Intraoperative injections arrest the action of fibroblast within post-traumatic 24 hours, and injections after administration of local anesthetics could eliminate injection pain and physical challenge during injection due to the lesion density ${ }^{11,13}$. In Table $4^{6,9,14}$, the recurrence rates of the auricular keloids receiving intraoperative and postoperative intralesional corticosteroid injection ranged from $9.5 \%$ to $33.3 \%$. Despite long-lasting results, the complications comprised wound dehiscence, depigmentation, flap necrosis, and abscess formation at the intralesional site $e^{6,9,14}$. The keloid tissues treated with corticosteroid previously were adhesive to make complete surgical resection difficult, and thus there was no advantage to corticosteroid injection before scheduled excision ${ }^{15}$.

Irradiation could restore a balance between collagen degradation and synthesis by affecting extracellular matrix gene expression ${ }^{8}$. The recurrence rates of postoperative radiotherapy (Table 5$)^{16-20}$ varied from $4 \%$ to $19 \%$. Acute radiation-associated adverse events, including skin ulceration, delayed wound healing, and skin graft necrosis, as well as chronic complications including permanent color change and telangiectasia, were reported in these studies ${ }^{16-21}$. Radiation-based treatments for keloids consist of electrons, X-rays, and brachytherapy ${ }^{22}$. Although brachytherapy has the lowest recurrence rate, the radioactive source of brachytherapy (iridium-192) has not been widely available ${ }^{23}$. Electron beam irradiation was otherwise superior to X-ray in treating keloids ${ }^{24}$. In addition to radiation modality, radiation dosage, fractionation, and overall treatment time also determine the therapeutic effect ${ }^{25-29}$. There is a dose-response relationship in treating postoperative keloids, and the total dosage of $20 \mathrm{~Gy}$ to $30 \mathrm{~Gy}$ was suggested to achieve the lowest recurrence rate ${ }^{25-28}$. In our analyses, higher radiation dosage was associated with a lower recurrence rate $(p=0.001)$.

Nevertheless, there was a correlation between adverse effects and the total irradiation dose $\mathrm{e}^{25-28}$. An optimal dose to reach a balance between local control and adverse effects for resected auricular keloids has not been well-established. We proposed a radiation regimen of $15 \mathrm{~Gy}$ or $18 \mathrm{~Gy}$ in three fractions completed within two or four days, enabling the transition of keloid fibroblasts from radioresistant status into the radiosensitive status and normal skin cells to repair ${ }^{29}$. Skin cancers arising from the post-radiation auricular keloids have not been reported in our and other studies.

The multimodal approach in our study possesses the advantages of intralesional corticosteroid injection and radiotherapy to achieve a better recurrence control without additional complications. There was no delayed wound healing in a single session of intralesional injection. Routine postoperative injections were abandoned to reduce the side effects of long-term intralesional corticosteroid injections, including skin and subcutaneous fat atrophy, telangiectasia, pigmentary change, skin necrosis, and ulceration. In our study, there were no radiation-related complications, especially radiation-induced hyperpigmentation, which may be partly attributed to additional intraoperative corticosteroid injection, effective in treating radiation dermatitis ${ }^{30}$. Another similar study utilizing individualized surgery (simple excision, core excision with scar flap reconstruction, and complete keloid excision with adjacent flap plasty) following immediate corticosteroid injection and radiotherapy to treat 


\begin{tabular}{|c|c|c|c|c|c|c|c|c|c|c|c|c|c|}
\hline & $\begin{array}{l}\text { Patient } \\
\text { (N) }\end{array}$ & $\begin{array}{l}\text { Keloid } \\
(\mathrm{N})\end{array}$ & Ethnicity & $\begin{array}{l}\text { Age } \\
\text { (years) }\end{array}$ & Sex & Location & Prior OP & Surgery & $\begin{array}{l}\text { External } \\
\text { beam RT }\end{array}$ & $\begin{array}{l}\text { RT } \\
\text { regimen } \\
\text { (total } \\
\text { dose/ } \\
\text { fraction } \times \\
\text { dose per } \\
\text { fraction) } \\
\text { (Gy) }\end{array}$ & $\begin{array}{l}\text { Recurrence } \\
\text { rate (keloid } \\
\text { N) }\end{array}$ & $\begin{array}{l}\text { Follow-up } \\
\text { (months) }\end{array}$ & $\begin{array}{l}\text { Complication } \\
\text { (keloid N) }\end{array}$ \\
\hline $1^{16}$ & 23 & 30 & NA & 28 & $\begin{array}{l}\mathrm{F}(75 \%) \\
\mathrm{M}(35 \%)\end{array}$ & $\begin{array}{l}\text { Lobe } \\
(100 \%)\end{array}$ & $\begin{array}{l}33.3 \% \\
(10 / 30)\end{array}$ & $\begin{array}{l}\text { Total exci- } \\
\text { sion }\end{array}$ & $\begin{array}{l}\text { 6-MeV } \\
\text { electron }\end{array}$ & $\begin{array}{l}15 / 3 \times 5 ; 1 \\
\text { Fr. in post- } \\
\text { OP } 2 \mathrm{~h} \\
\text { and } 2 \mathrm{Fr} \text {. } \\
\text { in post-OP } \\
2-3 \text { days }\end{array}$ & $13.3 \%(4 / 30)$ & 26 & $\begin{array}{l}\text { Hyperpigmen- } \\
\text { tation } 13.3 \% \\
(4 / 30)\end{array}$ \\
\hline $2^{17}$ & 57 & 63 & NA & NA & $\begin{array}{l}\mathrm{F}(91.2 \%) \\
\mathrm{M}(8.8 \%)\end{array}$ & NA & $0 \%$ & $\begin{array}{l}\text { Total exci- } \\
\text { sion: } 59 \% \\
\text { (37/63) } \\
\text { Enucleation: } \\
41 \%(26 / 63)\end{array}$ & $\begin{array}{l}4-\mathrm{MeV} \\
\text { electron }\end{array}$ & $\begin{array}{l}15 / 3 \times 5 \\
\text { over post- } \\
\text { OP } 3 \text { days }\end{array}$ & $\begin{array}{l}4.8 \%(3 / 63) \\
(8.1 \% \text { for } \\
\text { total exci- } \\
\text { sion and } 0 \% \\
\text { for enuclea- } \\
\text { tion) }\end{array}$ & 18 & No \\
\hline $3^{18}$ & 35 & NA & NA & 24 & $\begin{array}{l}\mathrm{F}(97 \%) \\
\mathrm{M}(3 \%)\end{array}$ & $\begin{array}{l}\text { Lobe } \\
(100 \%)\end{array}$ & $\begin{array}{l}\text { All with } \\
\text { OP or IL }\end{array}$ & $\begin{array}{l}\text { Total exci- } \\
\text { sion }\end{array}$ & $\begin{array}{l}100 \mathrm{kV} \\
\mathrm{X}-\text { ray } \\
\text { photon }\end{array}$ & $\begin{array}{l}10 / 1 \times 10 \text {; } \\
\text { within } \\
\text { post-OP } \\
24 \mathrm{~h}\end{array}$ & $\begin{array}{l}11.8 \% \\
(4 / 34)^{\mathrm{a}}\end{array}$ & 60 & $\begin{array}{l}\text { Transient } \\
\text { erythema and } \\
\text { post-radiation } \\
\text { hyperpigmen- } \\
\text { tation }\end{array}$ \\
\hline $4^{19}$ & 145 & 174 & Japanese & NA & NA & $\begin{array}{l}\text { Lobe } \\
(100 \%)\end{array}$ & $\begin{array}{l}13 \% \\
(19 / 145)\end{array}$ & $\begin{array}{l}\text { Wedge exci- } \\
\text { sion + PS } \\
\text { Simple exci- } \\
\text { sion + PS } \\
\text { Simple exci- } \\
\text { sion + V-Y } \\
\text { flap }\end{array}$ & $\begin{array}{l}\text { 4-MeV } \\
\text { electron }\end{array}$ & $\begin{array}{l}\text { High dose: } \\
15 / 3 \times 5 ; \\
\text { over post- } \\
\text { OP } 3 \text { days } \\
\text { (20\%) } \\
\text { Low dose: } \\
10 / 2 \times 5 \text {; } \\
\text { over post- } \\
\text { OP } 2 \text { days } \\
(80 \%)\end{array}$ & $\begin{array}{l}4.0 \%(7 / 174) \\
(4.7 \% \text { for } \\
\text { primary } \\
\text { keloid and } \\
0 \% \text { for } \\
\text { recurred } \\
\text { keloid) }\end{array}$ & 18 & NA \\
\hline $5^{20}$ & 21 & NA & African & 22 & $\begin{array}{l}\mathrm{F}(77.5 \%) \\
\mathrm{M}(22.5 \%)\end{array}$ & $\begin{array}{l}\text { Lobe } \\
(72 \%) \\
\text { Helix } \\
(28 \%)\end{array}$ & $\begin{array}{l}33.3 \% \\
(7 / 21)\end{array}$ & $\begin{array}{l}\text { Total exci- } \\
\text { sion } \pm \text { rhom- } \\
\text { boid flap }\end{array}$ & $\begin{array}{l}100 \mathrm{kV} \\
\mathrm{X} \text {-ray } \\
\text { photon }\end{array}$ & $\begin{array}{l}12 / 3 \times 4 \text {; } \\
\text { each Fr. on } \\
\text { post-OP } \\
3 \text { rd, 4th, } \\
\text { and 5th } \\
\text { day }\end{array}$ & $\begin{array}{l}19.1 \% \\
(4 / 21)^{\mathrm{a}} \\
(14.3 \% \text { for } \\
\text { primary } \\
\text { keloid and } \\
28.6 \% \text { for } \\
\text { recurred } \\
\text { keloid) }\end{array}$ & 18 & $\begin{array}{l}\text { Depigmen- } \\
\text { tation and } \\
\text { atrophy after } \\
\text { IL (100\%), } \\
\text { infection } \\
\text { (1), and flap } \\
\text { necrosis (1) }\end{array}$ \\
\hline Our & 35 & 45 & $\begin{array}{l}\text { Chinese } \\
(97.1 \%) \\
\text { Caucasian } \\
(2.9 \%)\end{array}$ & 27.1 & $\begin{array}{l}\mathrm{F}(85.7 \%) \\
\mathrm{M}(14.3 \%)\end{array}$ & $\begin{array}{l}\text { Lobe } \\
(71.1 \%) \\
\text { Helix } \\
(28.9 \%)\end{array}$ & $\begin{array}{l}17.8 \% \\
(8 / 45)\end{array}$ & $\begin{array}{l}\text { Enuclea- } \\
\text { tion + core } \\
\text { fillet flap }\end{array}$ & $\begin{array}{l}\text { 6-MeV } \\
\text { electron }\end{array}$ & $\begin{array}{l}15 / 3 \times 5 \text { or } \\
18 / 3 \times 6 ; 1 \\
\text { Fr. in post- } \\
\text { OP } 24 \mathrm{~h} \\
\text { and } 2 \mathrm{Fr} \text {. } \\
\text { in post-OP } \\
2-4 \text { days }\end{array}$ & $6.7 \%(3 / 45)$ & 24.1 & $\begin{array}{l}2.2 \%(1 / 45) \\
\text { seroma }(1)\end{array}$ \\
\hline
\end{tabular}

Table 5. Comparison with previous literatures adopting surgical excision with postoperative radiation

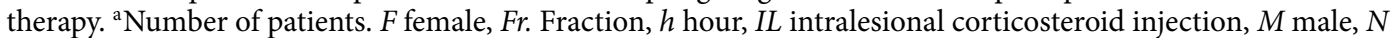
number, $N A$ not available, $O P$ operation, $P S$ primary suture, $R T$ radiation therapy.

auricular keloids showed a recurrence rate of $12.39 \%{ }^{31}$. Although a high total irradiation dose (20 Gy) and additional three postoperative monthly injections were applied, the recurrence rate was not superior to our results.

\section{Limitations}

This is a retrospective single center study, and further large-scale randomized controlled trial with an extended follow-up period is needed to confirm the superiority of this method compared to other conventional treatments. Despite these limitations, this novel multimodal approach can serve as a therapeutic option for auricular keloids.

\section{Conclusion}

In conclusion, we demonstrated that enucleation and core fillet flap followed by intraoperative intralesional corticosteroid injection and immediate postoperative radiotherapy obtained a low recurrence rate and minimal complications. This novel multimodal regimen in a single session is an effective and safe treatment for auricular keloids.

Received: 23 September 2021; Accepted: 4 February 2022

Published online: 03 March 2022

\section{References}

1. Yang, Y., Jiang, C. \& Xu, Q. Combination therapy for bulky auricular keloids: A clinical experience. J. Cosmet. Dermatol. 21, 14-16 (2019). 
2. Khansa, I., Harrison, B. \& Janis, J. E. Evidence-based scar management: How to improve results with technique and technology. Plast. Reconstr. Surg. 138, 165S-178S (2016).

3. Thornton, N. J., Garcia, B. A., Hoyer, P. \& Wilkerson, M. G. Keloid scars: An updated review of combination therapies. Cureus. 13, $12999(2021)$.

4. Park, T. H., Seo, S. W., Kim, J. K. \& Chang, C. H. Earlobe keloids: Classification according to gross morphology determines proper surgical approach. Dermatol. Surg. 38, 406-412 (2012).

5. Machol, J. A., Fang, R. C. \& Matloub, H. S. The free fillet flap after traumatic amputation: A review of literature and case report. Hand 8, 487-490 (2013).

6. Al Aradi, I. K., Alawadhi, S. A. \& Alkhawaja, F. A. Earlobe keloids: A pilot study of the efficacy of keloidectomy with core fillet flap and adjuvant intralesional corticosteroids. Dermatol. Surg. 39, 1514-1519 (2013).

7. Froelich, K., Staudenmaier, R., Kleinsasser, N. \& Hagen, R. Therapy of auricular keloids: Review of different treatment modalities and proposal for a therapeutic algorithm. Eur. Arch. Otorhinolaryngol. 264, 1497-1508 (2007).

8. Durani, P. \& Bayat, A. Levels of evidence for the treatment of keloid disease. J. Plast. Reconstr. Aesthet. Surg. 61, 4-17 (2008).

9. Sclafani, A. P., Gordon, L., Chadha, M. \& Romo, T. III. Prevention of earlobe keloid recurrence with postoperative corticosteroid injections versus radiation therapy a randomized, prospective study and review of the literature. Dermatol. Surg. 22, 569-574 (1996).

10. Shin, J. Y., Lee, J. W., Roh, S. G., Lee, N. H. \& Yang, K. M. A comparison of the effectiveness of triamcinolone and radiation therapy for ear keloids after surgical excision: A systematic review and meta-analysis. Plast. Reconstr. Surg. 137, 1718-1725 (2016).

11. Coppola, M. M., Salzillo, R., Segreto, F. \& Persichetti, P. Triamcinolone acetonide intralesional injection for the treatment of keloid scars: Patient selection and perspectives. Clin. Cosmet. Investig. Dermatol. 11, 387 (2018).

12. Atiyeh, B. S. Nonsurgical management of hypertrophic scars: Evidence-based therapies, standard practices, and emerging methods. Aesthetic Plast. Surg. 31, 468-492 (2007).

13. Emad, M., Omidvari, S., Dastgheib, L., Mortazavi, A. \& Ghaem, H. Surgical excision and immediate postoperative radiotherapy versus cryotherapy and intralesional steroids in the management of keloids: A prospective clinical trial. Med. Princ. Pract. 19, $402-405$ (2010).

14. Rosen, D. J., Patel, M. K., Freeman, K. \& Weiss, P. R. A primary protocol for the management of ear keloids: Results of excision combined with intraoperative and postoperative steroid injections. Plast. Reconstr. Surg. 120, 1395-1400 (2007).

15. Aluko-Olokun, B., Olaitan, A. A., Morgan, R. E. \& Adediran, O. M. Prevention of earlobe keloid recurrence after excision: Assessment of the value of presurgical injection of triamcinolone. J. Craniofac. Surg. 29, e673-e675 (2018).

16. Liu, C. L. \& Yuan, Z. Y. Retrospective study of immediate postoperative electron radiotherapy for therapy-resistant earlobe keloids. Arch. Dermatol. Res. 311, 469-475 (2019).

17. Ogawa, R. et al. Analysis of the surgical treatments of 63 keloids on the cartilaginous part of the auricle: Effectiveness of the core excision method. Plast. Reconstr. Surg. 135, 868-875 (2015).

18. Ragoowansi, R., Powell, B. W., Moss, A. L. H., Cornes, P. G. S. \& Glees, J. P. Ear-lobe keloids: Treatment by a protocol of surgical excision and immediate postoperative adjuvant radiotherapy. Br. J. Plast. Surg. 54, 504-508 (2001).

19. Ogawa, R. et al. Analysis of surgical treatments for earlobe keloids: Analysis of 174 lesions in 145 patients. Plast. Reconstr. Surg. 132, 818e-825e (2013).

20. Narakula, G. K. \& Shenoy, R. K. A prospective clinical review of "multi-model” approach for treating ear keloids. Indian J. Plast. Surg. 41, 2 (2008).

21. Shen, J. et al. Hypofractionated electron-beam radiation therapy for keloids: Retrospective study of 568 cases with 834 lesions. J. Radiat. Res. 56, 811-817 (2015).

22. Zainib, M. \& Amin, N. P. Radiation Therapy in the Treatment of Keloids (StatPearls, 2020).

23. Mankowski, P., Kanevsky, J., Tomlinson, J., Dyachenko, A. \& Luc, M. Optimizing radiotherapy for keloids: A meta-analysis systematic review comparing recurrence rates between different radiation modalities. Ann. Plast. Surg. 78, 403-411 (2017).

24. Maarouf, M., Schleicher, U., Schmachtenberg, A. \& Ammon, J. Radiotherapy in the management of keloids: Clinical experience with electron beam irradiation and comparison with X-ray therapy. Strahlenther. Onkol. 178, 330-335 (2002).

25. Flickinger, J. C. A radiobiological analysis of multicenter data for postoperative keloid radiotherapy. Int. J. Radiat. Oncol. Biol. Phys. 79, 1164-1170 (2011).

26. Kal, H. B. \& Veen, R. E. Biologically effective doses of postoperative radiotherapy in the prevention of keloids. Dose-effect relationship. Strahlenther. Onkol. 181, 717-723 (2005).

27. Renz, P. et al. Dose effect in adjuvant radiation therapy for the treatment of resected keloids. Int. J. Radiat. Oncol. Biol. Phys. 102, 149-154 (2018).

28. Sakamoto, T., Oya, N., Shibuya, K., Nagata, Y. \& Hiraoka, M. Dose-response relationship and dose optimization in radiotherapy of postoperative keloids. Radiother. Oncol. 91, 271-276 (2009).

29. Xu, J., Yang, E., Yu, N. Z. \& Long, X. Radiation therapy in keloids treatment: History, strategy, effectiveness, and complication. Chin. Med. J. 130, 1715-1721 (2017).

30. Assaf, H. A. R., El-Hamd, M. A., Nada, E. \& Marzouk, A. Clinical assessment of the efficacy and safety of intralesional injection of triamcinolone acetonide for treatment of chronic radio dermatitis. Arch. Biol. Biomed. Res. 1, 89-95 (2017).

31. Sun, Q., Zhou, Y., Tong, S., Zhou, K. J. \& Guo, S. Individualized surgery combined with radiotherapy and triamcinolone acetonide injection for the treatment of auricular keloids. BMC Surg. 21, 1-7 (2021).

\section{Author contributions}

Y.T.H., S.M.L., and C.Y.N. contributed to the conception and design of the study; Y.T.H., S.M.L., I.S.T., and C.Y.N. analyzed the data; and Y.T.H., S.M.L., and C.Y.N. contributed to the writing of the manuscript. All authors critically reviewed the manuscript and approved the final version.

\section{Competing interests}

The authors declare no competing interests.

\section{Additional information}

Correspondence and requests for materials should be addressed to C.Y.N.

Reprints and permissions information is available at www.nature.com/reprints.

Publisher's note Springer Nature remains neutral with regard to jurisdictional claims in published maps and institutional affiliations. 
(c) (i) Open Access This article is licensed under a Creative Commons Attribution 4.0 International cc) License, which permits use, sharing, adaptation, distribution and reproduction in any medium or format, as long as you give appropriate credit to the original author(s) and the source, provide a link to the Creative Commons licence, and indicate if changes were made. The images or other third party material in this article are included in the article's Creative Commons licence, unless indicated otherwise in a credit line to the material. If material is not included in the article's Creative Commons licence and your intended use is not permitted by statutory regulation or exceeds the permitted use, you will need to obtain permission directly from the copyright holder. To view a copy of this licence, visit http://creativecommons.org/licenses/by/4.0/.

(C) The Author(s) 2022 